\title{
AC Followed by Docetaxel Regimen
}

National Cancer Institute

\section{Source}

National Cancer Institute. AC Followed by Docetaxel Regimen. NCI Thesaurus. Code C138030.

A chemotherapy regimen consisting of cyclophosphamide and doxorubicin followed by docetaxel, used as a neoadjuvant or adjuvant treatment for breast cancer. 